\title{
Recién nacido en etapa terminal. ¿Qué decide el especialista desde el punto de vista bioético?
}

\author{
Eduardo E. Carsi-Bocanegra* \\ Departamento de Pediatría, Hospital Ángeles Pedregal, Ciudad de México, México
}

\section{Resumen}

Introducción: La bioética se ha ido posicionando con mayor fuerza en los últimos años, aunque se estima que falta mucho por hacerse. Se requieren consensos, capacitación y reforzar un marco jurídico Nacional que dé soporte legal a determinadas decisiones de los neonatólogos, en comunión con los padres del recién nacido, cuando este se encuentra en estado crítico y en etapa terminal. Material y métodos: Estudio transversal basado en una encuesta enviada vía electrónica a varios pediatras y neonatólogos del país. Resultados: Entienden los conceptos de ortotanasia, eutanasia, limitación del esfuerzo terapéutico y distanasia, el 66.8, 80.1, 78.2 y 82.8\% de los participantes, respectivamente. El 55.4\% ha negado el ingreso a neonatos con malformaciones mayores y el $70.9 \%$ explicaron su decisión a los padres; un $73.8 \%$ ha llevado a cabo limitación del esfuerzo terapéutico; un $81.2 \%$ ha dado órdenes de no reanimar y un $73.8 \%$ rechazan tratamientos fútiles. Aunque la mayoría habla con los padres, de éstos solo el $69 \%$ piden que se firme el consentimiento informado. Casi el $40 \%$ no cuenta con comités de bioética y solo el $31.3 \%$ de los que lo tienen solicita su intervención. Al $87 \%$ les gustaría que se legislara sobre ortotanasia y limitación terapéutica. Discusión: Un número significativo acepta y toma decisiones en torno a la ortotanasia pero hay una proporción importante que no habla con los padres o no pide la firma del consentimiento informado, lo que los expone a situaciones legales problemáticas. Conclusión: Existe una conciencia bioética del pediatra y el neonatólogo que trabajan en cuidados intensivos pero falta mejorar la relación médico-familiares y protegerse legalmente.

Palabras clave: Recién nacidos. Bioética. Relación médico-familiares. Consentimiento informado.

\section{Newborn in terminal stage. Which are the bioethical specialist decisions?}

\section{Abstract}

Introduction: In the last years, bioethics has positioned itself stronger, although there is a lot of work to do. Consensus, training, and a better legal National framework are required, to give strength for decision making of neonatologists, in agreement with parents of very sick, critical terminal ill infants. Material and methods: Transversal research based in an electronic poll sent to Neonatologist and Pediatricians of the country. Results: Concepts like orthotanasia, euthanasia, limitation for the therapeutic effort and dysthanasia are well understood in 66.8, 80.1, 78.2 and 82.8\%, respectively. 55.4\% of participants has denied the income to an intensive neonatal care unit to babies with major malformations and $70.9 \%$ of them talked of this with parents; $73.8 \%$ has decided limitation for the therapeutic effort; $81.2 \%$ has settled orders for no reanimation and $73.8 \%$ do not agree with futile treatments. Although the majority talked with parents, of these just $69 \%$ requested to sign an informed consent. Almost $40 \%$ do not have a Bioethics Committee in their workplace and among those who have it, just $31.3 \%$ involves

\section{Correspondencia:}

${ }^{*}$ Eduardo E. Carsi-Bocanegra

E-mail: carsipediatra@ hotmail.com
Fecha de recepción: 01-10-2018

Fecha de aceptación: 12-11-2018

DOI: 10.24875/PER.M19000002
Disponible en internet: 25-03-2019 Perinatol Reprod Hum. 2019;33:3-13

www.perinatologia.mx

0187-5337/C 2018. Instituto Nacional de Perinatología Isidro Espinosa de los Reyes. Publicado por Permanyer México SA de CV. Este es un artículo Open Access bajo la licencia CC BY-NC-ND (http://creativecommons.org/licenses/by-nc-nd/4.0/). 
them in their decisions. $87 \%$ of the specialists agree on the need for legal legislation in orthotanasia and limitation for therapeutic effort. Discussion: A significant number of professionals accepts and makes decisions about orthotanasia but there are many who still do not talk with the parents and neither do ask for the informed consent, which could result in legal problems. Conclusion: There is a bioethical awareness in Neonatologists and Pediatricians that work in neonatal intensive care units but it is urgent to improve the relationship between doctors and parents of babies and to protect themselves legally.

Key words: Newborn. Bioethics. Doctors-family relationship. Informed consent.

«La supervivencia de un niño gravemente dañado no debiera considerarse un éxito.» Mario Sebastiani y José María Ceriani ${ }^{1}$

\section{Introducción}

La ética es una rama de la filosofía que estudia la moral en el actuar del ser humano. Y la bioética, una palabra compuesta de dos palabras griegas, bios (vida) y ethikós (ética), se ocupa de la ética relacionada con la vida².

Beauchamp y Childress, en EE.UU., definieron los cuatro principios bioéticos, pilares en los que debe basarse el actuar del médico: autonomía, beneficiencia, no maleficencia y justicia ${ }^{3}$.

En la práctica clínica, respecto al primer concepto, el paciente tiene el derecho a ser informado de su situación clínica (y, por ende, el médico la obligación de informarle), explicando las sospechas y los hallazgos, el plan diagnóstico pendiente, el plan de manejo terapéutico, los riesgos, el pronóstico, los beneficios potenciales y también los efectos potencialmente adversos, la calidad de vida esperada, las posibilidades de supervivencia, y entonces, poder decidir libremente y sin presiones si acepta el tratamiento y los procesos de estudio por venir, o no.

Por beneficencia se entienden todas aquellas acciones que el médico realizará para promover la salud del ser humano. No causar daño al paciente, lo que representa la no maleficencia, es el concepto quizá fundamental, pues se trata de evitar hacer daño aunque este no sea el fin. Y, finalmente, la justicia, es decir, la distribución equitativa de los recursos médicos entre todos y cada uno de los pacientes, dejando de lado la discriminación.

La bioética es una rama de la ética en la que debe basarse el personal de salud, para llevar a cabo las acciones necesarias que permitan un trato médico digno y respetuoso del ser humano.

Las consideraciones éticas en la medicina neonatal han cobrado fuerza, aunque se estima que falta mucho por hacerse. Se debe llegar a consensos y, sobre todo, al establecimiento de un marco jurídico Nacional que dé soporte legal a determinadas decisiones que tienen que tomar los neonatólogos y pediatras que laboran con recién nacidos, en comunión con los padres del recién nacido, cuando éste se encuentra en estado crítico y en etapa terminal.

Sin embargo, poco se sabe, en general, del actuar del médico neonatólogo, en situaciones de esta índole. Son escasos los centros hospitalarios o los grupos médicos que tengan establecido un protocolo de conducta encaminado a cuidar estos aspectos bioéticos.

También es casi nula la acción de los hospitales para dar soporte a decisiones difíciles donde pueda estar comprometida la ética.

Para poder tener una idea clara y actualizada de la forma en que piensa y/o actúa el neonatólogo o el pediatra que trabajen con neonatos en cuidados intensivos, se elaboró una encuesta para ser respondida libremente.

\section{Material y métodos}

Durante los meses de enero a marzo del 2018 se realizó un estudio transversal, cerrado, ciego al investigador, en el que se diseñó una encuesta que se subió a la plataforma https://www.onlineencuesta.com. Apoyándonos en una base de datos y en las redes sociales, se notificó e invitó a médicos pediatras y médicos neonatólogos del país que laboraran con neonatos en unidades de cuidados intensivos neonatales para que accediesen a la encuesta mediante el vínculo que les fue enviado. Para garantizar el anonimato y la libertad en las respuestas no se pidió ni el nombre ni el correo electrónico del encuestado y en la plataforma encuestadora se inactivó la casilla de identificación de acceso cada vez que ingresaba un médico a la encuesta.

Ésta estuvo compuesta de 14 reactivos. El primero, para investigar el grado de conocimiento de cuatro conceptos: eutanasia, ortotanasia, distanasia y limitación del esfuerzo terapéutico (LET) (Tabla 1). Como veremos en la discusión, el concepto de este último hoy en día ha virado a adecuación del esfuerzo terapéutico (AET). Pero dado que los especialistas no bioeticistas estamos aún más allegados al término LET, se mantuvo esta definición. 
Tabla 1. Grado de conocimiento de cuatro conceptos: eutanasia, ortotanasia, distanasia y limitación del esfuerzo terapéutico

\begin{tabular}{|c|c|}
\hline Concepto & Definición \\
\hline A. Eutanasia & $\begin{array}{l}\text { Implica la o las acciones deliberadas para terminar con la vida de un paciente con enfermedad grave e } \\
\text { irreversible. Está asociada a la muerte sin sufrimiento físico. }\end{array}$ \\
\hline B. Distanasia & $\begin{array}{l}\text { Ensañamiento u obstinación terapéutica para utilizar todos los medios posibles, con el fin de prolongar la } \\
\text { vida de manera artificial. }\end{array}$ \\
\hline $\begin{array}{l}\text { C. Limitación del } \\
\text { esfuerzo terapéutico }\end{array}$ & $\begin{array}{l}\text { No aplicar nuevas medidas terapéuticas que se consideren fútiles o retirarlas, en un paciente con mal } \\
\text { pronóstico para la supervivencia o pronóstico de mala calidad de vida. }\end{array}$ \\
\hline D. Ortotanasia & $\begin{array}{l}\text { Permitir que la muerte sobrevenga en el momento que tenga que ocurrir, disminuyendo el sufrimiento sin } \\
\text { modificar la evolución de la enfermedad. }\end{array}$ \\
\hline
\end{tabular}

Tabla 2. Preguntas del proceder bioético

Pregunta 1. ¿Alguna vez ha negado el ingreso a la UCIN a algún neonato con malformaciones congénitas mayores o con evidencia de daño irreversible en órganos vitales?

Pregunta 2. ¿Habló con los padres del bebé explicando los motivos que justificaron su proceder?

Pregunta 3. ¿Alguna vez se ha negado a dar una indicación de algún tratamiento que usted sabe de antemano que solo prolongaría las constantes vitales del paciente sin conseguir su recuperación?

Pregunta 4. ¿Alguna vez ha dado órdenes de no reanimar o impedido que las realicen a un neonato en etapa terminal?

Pregunta 5. ¿Alguna vez ha indicado retirar medicamentos que solo están siendo útiles para mantener las constantes vitales?

Pregunta 6. ¿Alguna vez ha platicado previamente con los padres del bebé para convenir con ellos la toma de decisiones de los tres conceptos anteriores?

Pregunta 7. En caso afirmativo a la pregunta anterior, ¿les ha solicitado que firmen un documento de consentimiento informado sobre las decisiones que se hayan concluido?

Pregunta 8. ¿Ha "discutido" con sus compañeros de Servicio y/o Jefe de Servicio medidas de limitación terapéutica y/u órdenes de no reanimación en caso de paro cardiorrespiratorio de un bebé?

Pregunta 9. ¿Si hay comité de bioética en el hospital, ha discutido con ellos decisiones de limitación del esfuerzo terapéutico y/o la ortotanasia, en casos de neonatos irrecuperables, en etapa terminal?

Pregunta 10. ¿Estaría de acuerdo en que a nivel federal y estatal esté legislada la limitación terapéutica y la ortotanasia en el neonato crítico en México?

Los trece restantes exploraron el actuar bioético del médico que trabaja con neonatos en unidades de cuidados intensivos neonatales y su pensamiento relacionado con ello.

Se evitó hacer un cálculo de muestra porque la invitación se mandó de manera abierta y sin poder tener una estimación de cuántas personas que cumplieran con los criterios la verían y se interesarían.

Una vez finalizada la encuesta (Tabla 2) se obtuvieron los porcentajes de respuesta a cada pregunta formulada.

\section{Resultados}

Participaron 267 médicos, aunque no todos respondieron todas las preguntas. El $62 \%$ de los sujetos participantes fueron de sexo femenino.
El $31.43 \%$ de los encuestados están entre los 38 y 47 años; el $24.49 \%$ son sujetos de entre 28 y 37 años; aproximadamente otra cuarta parte, $23.67 \%$, son personas de entre 48 y 57 años; el $14.29 \%$ tienen entre 58 y 65 años, y solo un $6.12 \%$ son adultos mayores de 65 años.

En la figura 1 se pueden ver las respuestas correctas e incorrectas y las abstenciones para cada concepto explorado. El $82.8 \%$ contestó correctamente para el concepto de distanasia, el $80.1 \%$ para eutanasia, el $78.2 \%$ para LET y el $66.8 \%$ para el de ortotanasia.

Los resultados a la pregunta ¿Alguna vez ha negado el ingreso a la unidad de cuidados intensivos neonatales (UCIN) a algún neonato con malformaciones congénitas mayores o con evidencia de daño irreversible en órganos vitales? se pueden observar en la figura 2. El $55.4 \%$ ha negado alguna vez el ingreso, un $43.8 \%$ dijeron que no y un $0.8 \%$ no contestaron. 




Figura 1. Respuestas a conceptos bioéticos $(n=267)$.

LET: limitación del esfuerzo terapéutico; NC: no contestaron.

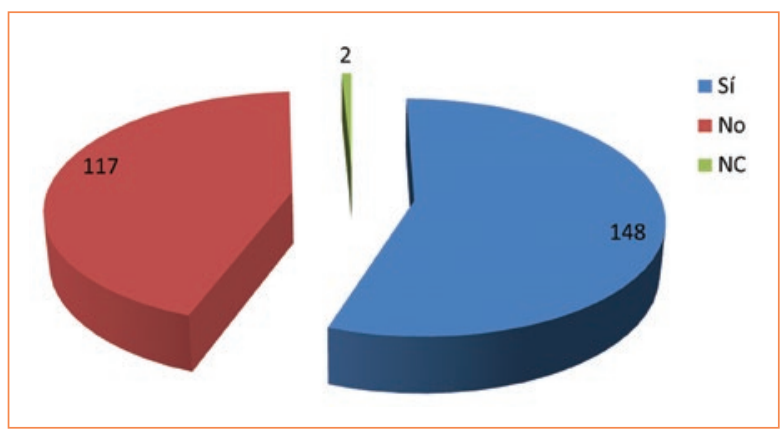

Figura 2. ¿Alguna vez ha negado el ingreso a la UCIN a algún neonato con malformaciones congénitas mayores o con evidencia de daño irreversible en órganos vitales? (n = 267).

NC: no contestaron.

La siguiente pregunta estaba ligada a los que aceptaron haber negado el ingreso a la UCIN a estos pacientes (148), para explorar la comunicación con los padres, lo que realiza el $70.9 \%$ de los médicos encuestados (Fig. 3).

Los resultados para la decisión relacionada con LET se pueden ver en la figura 4, donde en términos globales tres de cada cuatro sí lo ha hecho (73.8\%). Solo cuatro optaron por no contestar esta pregunta.

Para órdenes de no reanimar, la figura 5 nos indica que el $81.2 \%$ han decidido alguna vez no reanimar o han dejado esa instrucción dada, y solo tres médicos no contestaron.

Fue similar la respuesta para tratamientos fútiles, como se observa en la figura 6 , en donde el $72.6 \%$ de

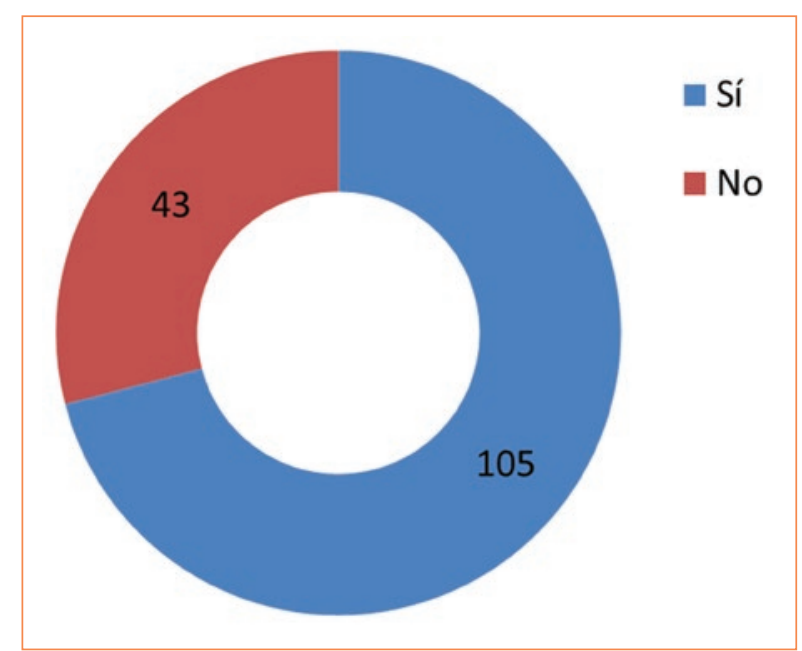

Figura 3. ¿Cuándo negó el ingreso a la UCIN para un recién nacido, habló con los padres del bebé explicando los motivos que justificaron su proceder?

los encuestados ha retirado medicamentos que consideran cumplen este punto.

Relacionado con hablar con los padres acerca de no indicar medicamentos o retirarlos por considerarlos fútiles o no reanimar en caso de paro cardíaco, cuyos resultados se observan en la figura 7 , el $79 \%$ aceptaron haber platicado con los padres. Cuatro no contestaron; sin embargo, solo 146 de 211 (69\%) solicitaron a los padres firmar un documento de consentimiento informado para tales decisiones.

Una mayoría, correspondiente al $83.5 \%$, discuten con el Jefe del Servicio y/o los colegas de este sobre 




Figura 4. ¿Alguna vez se ha negado a dar una indicación de algún tratamiento que usted sabe de antemano que solo prolongaría las constantes vitales del paciente sin conseguir su recuperación? ( $n=267)$. NC: no contestaron.

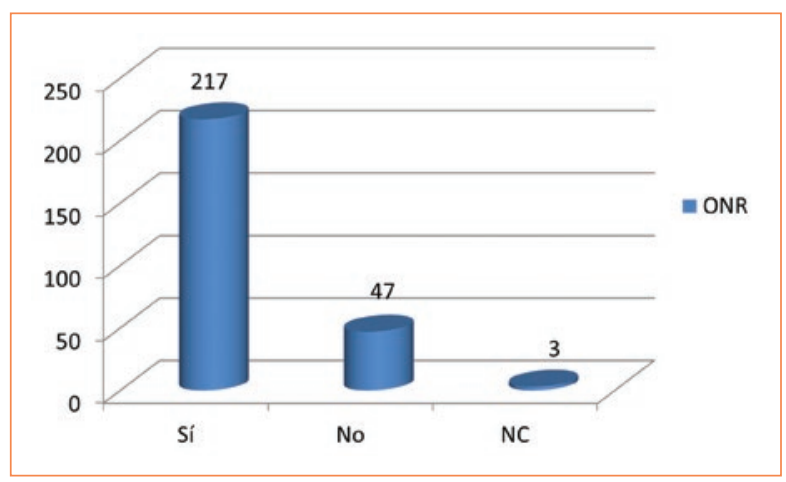

Figura 5. ¿Alguna vez ha dado órdenes de no reanimar 0 impedido que las realicen a un neonato en etapa terminal? ( $\mathrm{n}=267$ ).

ONR: orden de no reanimar; NC: no contestaron.

órdenes de LET o de no reanimar (Fig. 8). Nuevamente, cuatro médicos no contestaron.

En relación con un comité de bioética hospitalario, respondieron 252 participantes. De estos, solo el 31.4\% discuten con dicho comité asuntos relacionados con LET y ortotanasia; el $29.4 \%$ no lo hacen y el $39.2 \%$ refieren la inexistencia de este comité en el hospital donde laboran (Fig. 9).

Finalmente, en la figura 10 se observa el punto de vista sobre legislar en cuanto a LET u ortotanasia, aunque son conceptos ya contemplados en la Ley General de Salud. Hubo 22 médicos que no contestaron, pero los que están de acuerdo en legislar representan el $87 \%$.

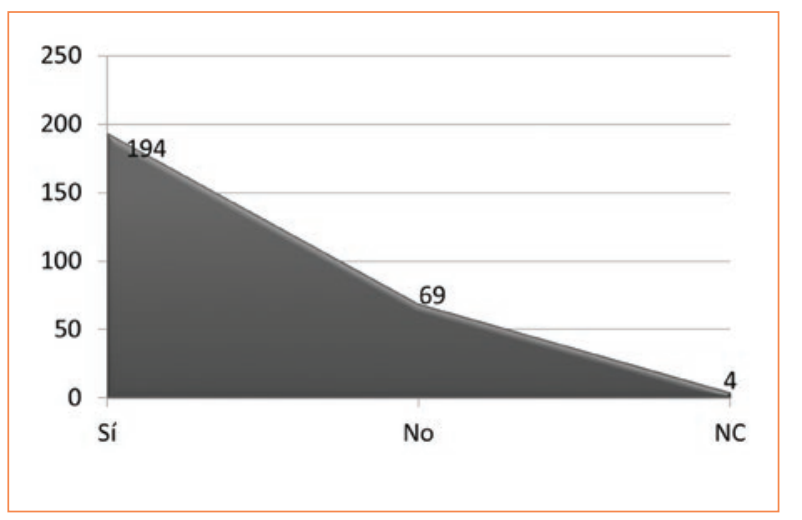

Figura 6. ¿Alguna vez ha indicado retirar medicamentos que solo están siendo útiles para mantener las constantes vitales? $(\mathrm{n}=267)$. NC: no contestaron.

\section{Discusión}

De acuerdo con las respuestas de la encuesta sobresale el actuar del médico en aspectos relacionados con la LET y las órdenes para no reanimar. Debo aclarar, empero, que hoy en día se ha modificado este término por el de AET. Desgraciadamente, muchos médicos que no somos bioeticistas desconocemos esta nueva terminología, pero vale la pena en el futuro inmediato actualizar conceptos, dada su importancia y la necesidad de modificaciones en el papel y en la práctica el manejo de un recién nacido en etapa terminal.

Limitar el esfuerzo deja un vacío tanto en el manejo médico como en la óptica de los padres del neonato. Limitar es ya no hacer algo cuando probablemente pudiese ser realizado. Adecuar, en cambio, significa dar un giro al plan terapéutico; aunque puede considerarse retirar algún tratamiento fútil o no iniciar otro que no va a resolver el problema, es voltear al manejo de soporte, al cambio de objetivos terapéuticos, que incluyan la sedación y la analgesia profunda; el no abandono del paciente y el apoyo psicológico, en este caso a los padres, para el corto tiempo que se espera de una etapa terminal; mantener la hidratación del paciente, por ejemplo. Al adecuar, pues, si bien se suspenden algunas medidas terapéuticas, aumentan otras que fortalezcan nuestra conducta humanista de limitar el sufrimiento del paciente y los padres ${ }^{4-7}$.

Como dice Pérez ${ }^{4}$, «preparar al paciente a una buena muerte». Y como también establece, no debe importarnos cuánto deterioro haya en el paciente; su dignidad se preserva intacta y por ende la medicina 


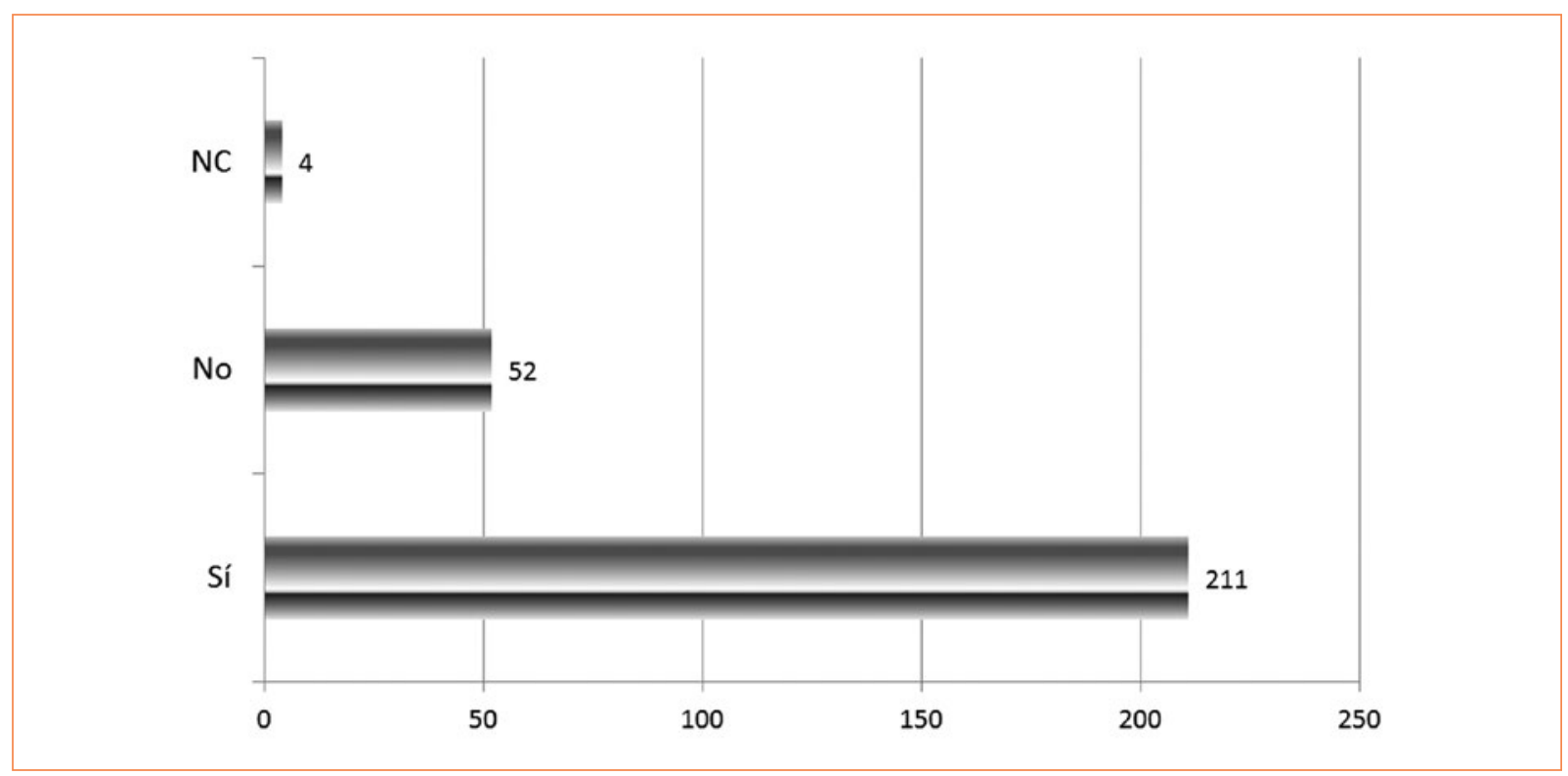

Figura 7. ¿Alguna vez ha platicado previamente con los padres del bebé para convenir con ellos la toma de decisiones de los tres conceptos anteriores? $(n=267)$.

NC: no contestaron.

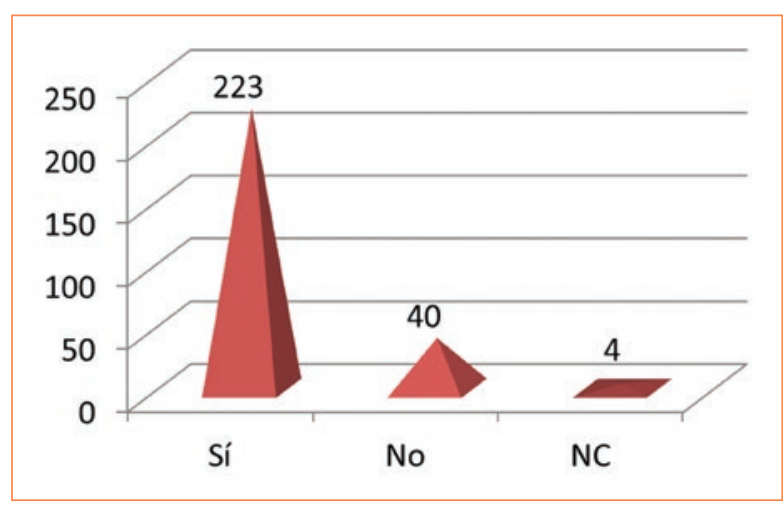

Figura 8. ¿Ha "discutido" con sus compañeros de Servicio y/o Jefe de Servicio medidas de limitación terapéutica y/u órdenes de no reanimación en caso de paro cardiorrespiratorio de un bebé? $(n=267)$.

NC: no contestaron.

paliativa es indispensable. $Y$ agregaría, no solo las medidas paliativas al paciente; sino convertir las unidades neonatales en un escenario más humanista, permitiendo a los padres estar presentes en ese final de vida de su hijo o hija. Permitir el contacto físico y respetarles ese momento de morir ${ }^{8}$.

Es importante considerar el tiempo transcurrido entre haber hablado con los padres sobre LET y el retiro del soporte vital para llegar a la AET. Puede variar desde

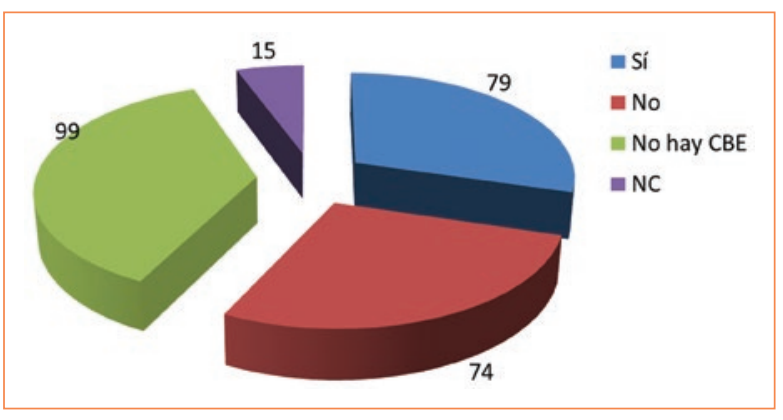

Figura 9. Si hay comité de bioética en el hospital, ¿ha discutido con ellos decisiones de limitación del esfuerzo terapéutico $\mathrm{y} / \mathrm{o}$ la ortotanasia, en casos de neonatos irrecuperables, en etapa terminal? $(n=267)$. CBE: comité de bioética; NC: no contestaron.

1 a 20 días, en función del tiempo que se lleva conversar con los padres y decidir éticamente que no hay más por hacer. Puede ser también el tiempo que en algunos casos se requiera al comité de bioética. $\mathrm{Si}$ bien 20 días se antojan demasiados y vienen a suponer una prolongación del sufrimiento, habrá casos en que esto es inevitable y de ahí la importancia de una buena estrategia para la $\mathrm{AET}^{7}$.

Así ocurre ya en varias unidades de cuidados intensivos en EE.UU. y Europa, dejando a un lado actitudes prepotentes del personal de salud y respetando más la 


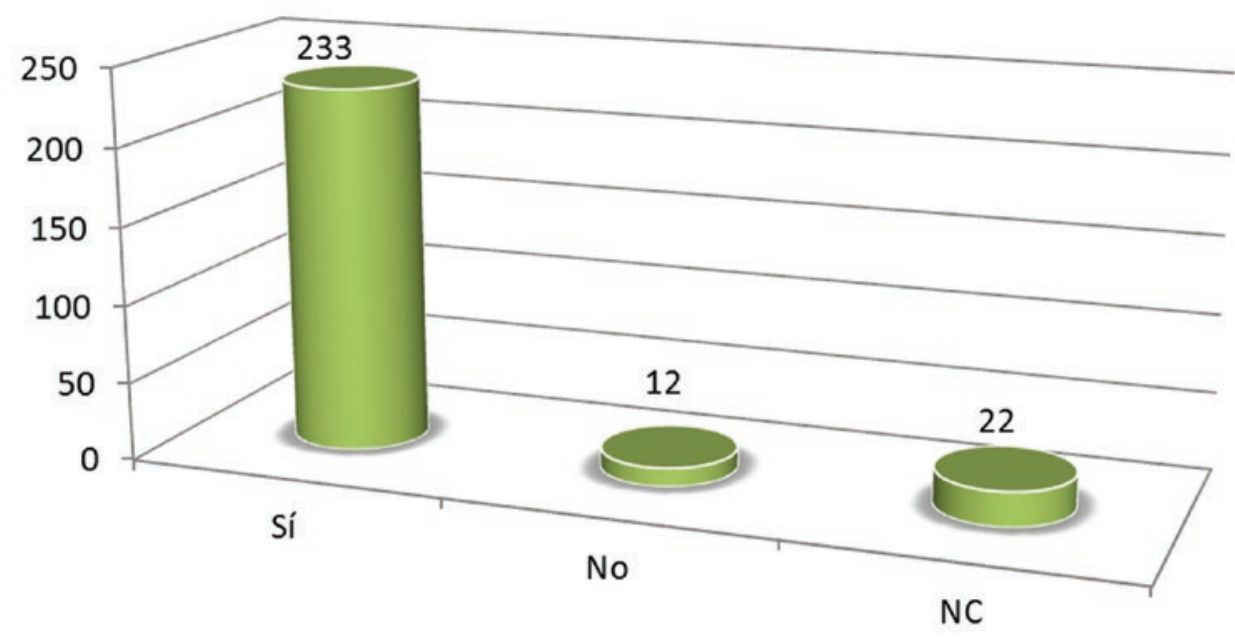

Figura 10. ¿Estaría de acuerdo en que a nivel federal y estatal esté legislada la limitación terapéutica y la ortotanasia en el neonato crítico en México? $(n=267)$.

NC: no contestaron.

autonomía del paciente, que en nuestro caso sería la de los padres del neonato. De manera práctica, estaríamos hablando de llevar a la práctica la ortotanasia.

Refiriéndonos a los cuatro conceptos fundamentales de la bioética, la AET está justificada porque evita la futilidad (justicia), la distanasia (no maleficencia), respeta la dignidad de la persona y reorienta la terapéutica para evitar el sufrimiento (beneficencia), así como respeta las voluntades de los padres en el caso de la neonatología (autonomía).

Desde una perspectiva de un análisis socialista de la bioética, Martínez ${ }^{9}$ analiza la evolución mundial de los cuatro principios básicos de la bioética y a partir de considerar que en su opinión hace falta hacer realidad los principios de justicia y autonomía, plantea la necesidad de un quinto principio, la solidaridad. Con éste se pueden potenciar los cuatro restantes, comenta.

La solidaridad nos permite hacer una introspección de nuestro actuar y hacer una causa común en tratar de evitar el sufrimiento del paciente y comprender y caminar con mayor peso, junto con los familiares, ante la eventualidad de una evolución fatal, dando paso así a la muerte digna del paciente, tan importante como vivir con dignidad.

La población encuestada quedó dividida en términos generales de manera armónica. $56 \%$ son médicos jóvenes y $44 \%$ son médicos maduros. Podría esperarse que los puntos de vista del médico mayor, por su propia formación, tuviese quizá un pensamiento no tan abierto a conceptos relativamente nuevos como la ortotanasia (el concepto de dolor en el neonato no era válido hasta hace algunos años). Sin embargo, en mi opinión es bueno ver que hay cierta uniformidad en criterios, independientemente de la edad de los profesionales encuestados.

Aunque se evidenció que hay un número de médicos que no tienen del todo claro los conceptos, la mayoría en general conoce los significados de eutanasia, distanasia, LET y ortotanasia. Y esto implicaría que estén más dispuestos a entender las situaciones críticas y tratar de favorecer un entorno más "armónico» dentro de los cuidados intensivos.

Por lo expuesto en sus respuestas, un número significativo ha puesto en práctica decisiones como no reanimar en casos específicos y, esperaría, bien fundamentados. También han realizado actos relacionados con la ortotanasia y la LET y ven con buenos ojos que se legisle en esos términos.

Es claro que los pediatras que trabajan en unidades de recién nacidos y los neonatólogos suelen enfrentarse a situaciones complejas en las cuales la toma de decisiones está «contaminada» con incertidumbres éticas. No es problema identificar al neonato grave, pero una vez identificado hay que delimitar el momento donde nuestros esfuerzos máximos para continuar luchando por la vida del paciente deben ahora reorientarse hacia un manejo que implique «lo mejor para el paciente». Pero, ¿bajo la perspectiva de quién es «lo 
mejor para el paciente»? ¿Realmente es una etapa terminal? ¿Estamos ante una enfermedad irreversible? ¿La calidad de vida verdaderamente será muy mala en caso de supervivencia?

La medicina y la ética se basan en una lógica de probabilidad, no en una lógica de certeza absoluta. Es aquí justo donde, basándonos en el manejo razonable de una incertidumbre, debemos acercarnos a quienes van a «ejercer la autonomía» del neonato, es decir, los padres, y deliberar con ellos. Arnaez, et al. ${ }^{10}$ puntualizan esta herramienta de deliberación, como "seguir una secuencia ordenada de actuación» deliberando sobre los hechos, los valores en conflicto y los cursos que habrán de seguir diversas acciones, así como cuáles son las consecuencias esperadas una vez que se tomen las decisiones.

De esta forma, sentarse con los padres para hablar sobre la condición aguda o crónica, crítica o terminal del paciente; explicar lo que se espera sobre la vida y calidad de vida del enfermo en el futuro inmediato pero más aún a largo plazo; los objetivos de los tratamientos propuestos: los terapéuticos y los paliativos; explicar cuándo un manejo es fútil y distinguir si el beneficio potencial sería solo para el órgano, aparato o sistema a tratar o para el paciente en general. En este proceso de deliberación, la comunicación del profesional de salud con los padres debe ser tanto de manera verbal como no verbal. Tan importante es la actitud compasiva, saber escuchar, saber acercarse a los padres, buscar el contacto ocular, respetar los silencios, identificar los sentimientos y los códigos de comunicación, como hablar con los padres al mismo tiempo, utilizando el nombre propio del hijo, sin palabras técnicas, sin desatender los cuestionamientos, ser honestos con la información, ser respetuosos, dejar un margen aunque sea pequeño a la esperanza y realizar la comunicación en privado ${ }^{10}$.

Cuando es inminente el deceso del neonato, se deben construir (rápidamente) puentes de comunicación con los padres, manteniéndolos bien informados y acompañarlos en el momento. Algunos preferirán privacidad, otros necesitarán el acompañamiento del personal de salud. Mientras tanto, el médico debe asegurar el control del dolor y favorecer la "comodidad" del neonato durante este proceso de agonía. Y cuando hay desacuerdos internos del grupo multidisciplinario en cuanto a las medidas a llevar a cabo o, con mayor razón, desacuerdos con los familiares, será indispensable la actuación del equipo de bioética del hospital para tomar las mejores decisiones. Cada unidad de terapia intensiva debe tener un protocolo, que sea claro y facilite al personal de salud la toma de estas decisiones. "Afrontando el sufrimiento, se hacen mejores médicos ${ }^{11}$.

El humanismo comprende, por una parte, la concepción del hombre como un todo, el cual mediante su capacidad de sentir y de pensar puede no solo entender el mundo en su totalidad, sino reflexionando y utilizando su intelecto, es también capaz de transformarlo; comprende asimismo lo que cada persona posee en esencia, la absoluta libertad para elegir y decidir sobre su vida, sin dejar de contextualizar a los demás, es decir, las relaciones sociales. A partir de esto, la ética dentro de la medicina y la relación médico-paciente se entiende ahora como equipo de salud (antes solo el médico) y el «usuario» (antes enfermo), quienes crean una interrelación, ya sea como promoción y prevención de la salud o para la recuperación de esta ${ }^{12}$.

En los casos graves, se trata de un equipo multidisciplinario que debe estar compuesto por un grupo sanitario tan diverso como el personal médico, de enfermería, de trabajo social, psicología, psiquiatría y tanatología, sin olvidar el apoyo espiritual que requiera el paciente o familiar del paciente.

Además, hay que asegurarse de que el paciente 0 sus familiares comprendan bien la situación que les aqueja, que estén tan bien informados que puedan participar en la toma de decisiones, que sientan tener la libertad de manifestarse a favor 0 en contra de la propuesta del equipo sanitario.

En opinión de Grzona ${ }^{8}$ hay diferencias en los criterios para realizar una LET en EE.UU., donde se fortalece la idea de "esperar la certeza», manteniendo así tratamientos intensivos hasta tener certeza de una muerte inminente, mientras que en Europa y de manera semejante en Latinoamérica predomina el criterio de un pronóstico individualizado donde se aplica inicialmente un tratamiento con máximos esfuerzos, seguido de una reevaluación del pronóstico que permita evitar la distanasia.

Empero, en Francia, por ejemplo, prevalece un concepto paternalista, al menos hasta hace poco, donde el médico no toma en cuenta a los padres en una decisión de retiro de medicamentos y en EE.UU. tienden a no consultarlos para saber si continúan o no, así como en las órdenes de no reanimar ${ }^{13}$.

En cuanto a los comités de bioética, Jasso ${ }^{14}$, en su excelente apunte de la evolución mundial de la bioética en los neonatos, puntualiza la necesidad de estos comités donde exista una Unidad de Neonatología. Y menciona los puntos finos a partir de los que debe trabajar este equipo: identificar las circunstancias 0 
factores externos o internos que pueden influir en el proceso; identificar y describir los problemas; establecer desde el punto de vista ético cuál es el bien que se busca; el cuestionamiento jurídico basado en saber si las acciones son o no legales; conocer la normativa del hospital y/o instituto donde se labora y estar seguro de que se sigue; jerarquizar los problemas éticos como la proporcionalidad de la terapéutica implementada y definir el bien que se busca, todo en torno al paciente, pero también a los familiares y bajo qué circunstancias se actúa; definir las decisiones que pueden y deben tomarse, evitando la distanasia y, finalmente, las recomendaciones a través del discernimiento, apunta.

Existe una gran responsabilidad del médico para tomar decisiones difíciles en circunstancias complejas. Lo que el médico piensa y decide está también directamente relacionado con factores que inciden a lo largo de su vida y de su formación científica: la educación familiar, su nivel educativo, su nivel cultural, sus propias creencias religiosas, el entorno social que le rodea, los maestros que le han dejado huella, los intereses de la comunidad en la que habita y, por supuesto, las normas legales que rigen. Evitar la frustración y el dolor propios que impidan nuestro buen actuar razonado y juicioso, pero sin caer en la insensibilidad ${ }^{15}$.

Nuestra realidad, el pensamiento médico latinoamericano, es muy diferente a la de países europeos del primer mundo. En Francia es común la eutanasia, así como, en menor grado, en Holanda. Existe mucha variabilidad en conceptos como la LET, incluidos órdenes de no reanimar o suspender o no indicar tratamientos que varían entre el 46 y el $90 \%$ de acuerdo con el país europe $0^{16}$. Al parecer nuestra muestra refleja un pensamiento más homogéneo, si bien es solo una muestra de un solo país.

Es importante destacar que los familiares pasarán por diversas etapas de estrés, de negación, enojo y depresión, para finalmente llegar a la aceptación del hecho y el duelo final hasta la superación.

Hoy en día el médico tiene a su alcance un arsenal de equipo médico, de tecnología avanzada y medicamentos, que pueden mantener la «vida», aunque no haya esperanza de revertir el proceso morboso y mantener solo las constantes vitales del paciente aunque éste haya perdido el estado de alerta. Como cita Aréchiga ${ }^{17}$, el mandato primario dictado por Hipócrates era conservar la vida del paciente. Pero como bien apunta, hoy en día se exige también una muerte digna $y$ ante ello expone los conceptos de eutanasia, suicidio asistido y muerte por supresión del tratamiento médico. En nuestro país, la eutanasia no es legal, pero sí la ortotanasia. Por ello las «baterías» para el buen actuar bioético del médico deben dirigirse a este punto.

Hay varias organizaciones y sociedades médicas que han desarrollado guías de cómo abordar el manejo en neonatos considerando, además de sus condiciones clínicas su edad gestacional, y en donde se consideran todos los esfuerzos iniciales para neonatos con edad gestacional mayor de 25 semanas, medidas paliativas para los menores de 22 semanas e individualizar el plan terapéutico, en común acuerdo con los padres para quienes se encuentran en esta etapa ${ }^{18}$. Esto no puede ser aplicable en nuestro país de manera general, pues nuestra realidad es diferente y los límites de viabilidad en México en centros de alta especialidad gira en torno de las 25 semanas. Además, nuestra reglamentación de la Ley General de Salud es muy clara en cuanto a obtener el consentimiento informado en todos los casos, independientemente de la edad gestacional.

La Ley General de Salud en su artículo 166 bis habla de garantizar una muerte natural en condiciones dignas; y posteriormente incluye la definición de conceptos como obstinación terapéutica, y la responsabilidad de los padres, tutor o responsable legal para la toma de decisiones cuando de un menor de edad se trata. $Y$ en octubre del 2013 se presentaron modificaciones para incluir la ortotanasia, quedando asentada como se explica a continuación ${ }^{19}$. Para los efectos del artículo 166 bis 1, se entenderá por:

- IV. Enfermo en etapa terminal. Es la persona que tiene un padecimiento y/o enfermedad incurable, irreversible y mortal, y que por caso fortuito o causa de fuerza mayor tiene una esperanza de vida inferior a seis meses y se encuentra imposibilitado para mantener su vida de forma natural, con base en las siguientes circunstancias:

a) Presenta diagnóstico de enfermedad avanzada, irreversible, progresiva, incurable y/o degenerativa.

b) Imposibilidad de respuesta a tratamiento específico, $y / 0$

c) Presencia de numerosos problemas y síntomas secundarios o subsecuentes.

- VI. Ortotanasia significa muerte correcta. Distingue entre curar y cuidar, sin provocar la muerte de manera activa, directa o indirecta, evitando la aplicación de medios, tratamientos y/o padecimientos médicos obstinados, desproporcionados o inútiles, procurando no menoscabar la dignidad del enfermo en etapa terminal, otorgando los cuidados paliativos, las medidas mínimas ordinarias y teratológicas y, en su caso, la sedación controlada. 
- VII. Sedación controlada. Es la administración de fármacos por parte del personal de salud correspondiente para lograr el alivio inalcanzable son otras medias(sic), de un sufrimiento físico y/o psicológico, en un paciente en etapa terminal, con su consentimiento explícito, implícito, o delegado, sin provocar con ello la muerte de manera intencional de este.

En esta encuesta, un número importante de médicos no discute con los padres las decisiones en cuanto a tratamientos o retiro de los mismos. Menos aún solicitan la firma de un consentimiento informado y solo algunos buscan al comité de bioética, cuando lo hay. Esto es indebido y los deja en situaciones legales problemáticas.

Destaco el artículo 166 bis 20 de la Ley General de Salud: «El personal médico que, por decisión propia, deje de proporcionar cualquier tratamiento o cuidado sin el consentimiento del enfermo en situación terminal, o en caso de que esté impedido para expresar su voluntad, el de su familia o persona de confianza, será sancionado conforme lo establecido por las leyes aplicables".

$Y$ en cuanto a los comités de bioética, estos deben existir en los hospitales de nivel III. Es responsabilidad de las autoridades el velar por que estén debidamente formados, pero también es importante que el equipo de salud de un hospital lo exija.

El presente artículo tiene varias limitaciones. En primer lugar, el número reducido de participantes. En segundo término, se limitó la encuesta a un solo miembro del equipo de salud, el médico. En tercer lugar, aunque se invitó a pediatras y neonatólogos, se desconoce cuántos son pediatras y cuántos neonatólogos.

Finalmente, aunque siguen publicándose artículos con los conceptos de LET, deberemos en el futuro inmediato reorientarnos a la AET.

\section{Conclusiones}

En términos generales, existe un buen entendimiento del significado de conceptos como eutanasia, distanasia, ortotanasia y LET.

Falta concienciar a los directivos de hospitales de que se debe contar siempre con un comité de bioética.

El médico tratante debe mejorar su relación con los familiares o responsables legales del neonato, involucrarlos en la toma de decisiones dirigidas a ortotanasia y LET/AET, y solicitar la firma del consentimiento informado. Asimismo, debe exigir e involucrar al comité de bioética del hospital en esta difícil toma de decisiones.
Hace falta el trabajo multidisciplinario en torno a estos conceptos del paciente en etapa terminal.

\section{Agradecimientos}

Agradezco a todos los neonatólogos y pediatras que amablemente participaron contestando la encuesta.

\section{Financiamiento}

La presente investigación no ha recibido ayudas específicas provenientes de agencias del sector público, sector comercial o entidades sin ánimo de lucro.

\section{Conflicto de intereses}

Los autores declaran que no existe conflicto de intereses.

\section{Responsabilidades éticas}

Protección de personas y animales. Los autores declaran que para esta investigación no se han realizado experimentos en seres humanos ni en animales.

Confidencialidad de los datos. Los autores declaran que en este artículo no aparecen datos de pacientes.

Derecho a la privacidad y consentimiento informado. Los autores declaran que en este artículo no aparecen datos de pacientes.

\section{Bibliografía}

1. Sebastiani M, Ceriani JM. Aspectos bioéticos en el cuidado de los recién nacidos extremadamente prematuros. Arch Argent Pediatr. 2008;106: 242-48.

2. Andorno R. Bioética y dignidad de la persona. 2. ${ }^{a}$ ed. Madrid: Editorial Teknos; 2012

3. Beauchamp TL, Childress JF. Principios de ética biomedical. 4. a ed. Barcelona: Masson; 1999.

4. Pérez Pérez PF. Adecuación del esfuerzo terapéutico, una estrategia al final de la vida. Semergen. 2016;42(8):566-74

5. Bernardá M. Limitación/adecuación del esfuerzo terapéutico en Pediatría Arch Pediatr Urug. 2015;86:121-5.

6. Bátiz J. Adecuación del esfuerzo diagnóstico y terapéutico en cuidados paliativos. Gac Med Bilbao. 2014;111:57-62

7. Morales G. Limitación del esfuerzo terapéutico en cuidados intensivos pediátricos. Rev Chil Pediatr. 2015;86:56-60.

8. Grzona ME. Muerte digna en Neonatología. Rev Med Univ. 2008;4:1-12.

9. Martínez GJA. La bioética y los enfoques del final de la vida. Revista Cubana de Salud Pública. 2010;36(4)353-7.

10. Arnaez J, Tejedor JC, Caserío S, Montes MT, Moral MT, González DJ, et al. La bioética en el final de la vida en neonatología. Cuestiones no resueltas. An Pediatr (Barc). 2017;87(6):356.e1-356.e12.

11. Riani-Llano N. Momentos claves para humanizar el paso por la Unidad de Cuidados Intensivos Neonatales. Pers Bioét. 2007;11:138-45.

12. Ocampo MJ. La bioética y la relación médico paciente. Cir Cir. 2002;70: 55-9.

13. Orfali K. Parental role in medical decision making: fact or fiction? A comparative study of ethical dilemmas in French and American neonatal intensive care units. Soc Sci Med. 2004;58:2009-22. 
14. Jasso GL, Gamboa MJD. Evolución, impacto y aplicación de la bioética en el recién nacido. Bol Med Hosp Infant Mex. 2010;67:204-22.

15. Alonso Uría RM, Magraner Tarrau ME, Rodríguez Alonso B, Pupo Portal L. La toma de decisiones complejas en las unidades de cuidados intensivos neonatales. Rev Cubana Obstet Ginecol. 2006;32(2).

16. Cuttini M, Nadai M, Kaminski M, Hansen G, de Leeuw R, Lenoir S, et al. End-of-life decisions in neonatal intensive care: physicians' self-reported practices in seven European countries. EURONIC study group. Lancet. 2000;355:2112-8.

17. Aréchiga $\mathrm{H}$. La bioética y la formación científica del médico. Gac Med Mex. 2001;137:375-86.
18. Pignotti MS, Donzelli G. Perinatal care at the threshold of viability: an international comparison of practical guidelines for the treatment of extremely preterm births. Pediatrics. 2008;121:e193-8.

19. Ley General de Salud. Iniciativa que reforma los artículos 166 Bis y 166 Bis 1 de la Ley General de Salud, a cargo de Ricardo Mejía Berdeja y suscrita por Ricardo Monreal Ávila, diputados del Grupo Parlamentario de Movimiento Ciudadano [Internet]. Estados Unidos Mexicanos: Gaceta Parlamentaria [fecha de consulta: 04-05-18]. Disponible en: http://gaceta. diputados.gob.mx/Black/Gaceta/Anteriores/62/2013/oct/20131029-VIII/ Iniciativa-12.html. 